\title{
Energy and atomic number scan in electron-ion collisions
}

\section{George Prokhorov}

JINR, 141980 Dubna, Russia

E-mail: prokhorovatheor.jinr.ru

\section{Oleg Teryaev*}

JINR, 141980 Duvbna, Russia

E-mail: teryaevetheor.jinr.ru

\section{Valentin Zakharov}

Institute of Theoretical and Experimental Physics, NRC Kurchatov Institute, 117218 Moscow, Russia

School of Biomedicine, Far Eastern Federal University, 690950 Vladivostok, Russia Moscow Institute of Physics and Technology, 141700 Dolgoprudny, Russia

E-mail: vzakharov@itep.ru

XXVII International Workshop on Deep-Inelastic Scattering and Related Subjects - DIS2019 8-12 April, 2019

Torino, Italy

${ }^{*}$ Speaker. 


\section{Introduction}

Heavy ions provide the unique laboratory to test QCD at extreme conditions. The recent progress includes the particular impact on polarization generated by axial anomaly providing the robust explanation of energy dependence [1]. The non-central heavy-ion collisions (HIC) naturally provide the large angular momentum (defined by the impact parameter) and related vorticity, as a source of polarization. Vorticity and helicity calculations in kinetic model allowed to predict [2] and elaborate (see [3], where the role of gravitational anomaly in lattice simulations was addressed, and Ref. therein) the polarization in HIC, with the special attention to NICA complex at JINR.

At the same time, collisions of electrons with ions at EIC, due to point-like nature of electron, do not manifest (in a simple way) the appearance of impact parameter, while the role of acceleration should be more pronounced.

Here we discuss the special role of energy scan at EIC, having in mind also the lower energy EIC which might be also constructed in future as an extension of NICA. The decreasing temperature and possible manifestation of Unruh effects in acceleration is discussed in Section 2.

From the other side, the polarization for light ions and, at extreme, for hadrons, requires to consider the matching with the respective mechanisms of polarization generation. Here we point out for the first time to the similarity of Sivers function to the "memory" effect discussed earlier in string theory and recently suggested in QCD at high energies [4].

\section{Acceleration and Unruh radiation}

In collisions of heavy ions, as well as in processes with elementary particles, such as $e^{+} e^{-}$ annihilation, ultrahigh accelerations can occur. One of the mechanisms for generating big accelerations was proposed in [5] and is based on considering the string tension in the process of hadronization. Thus, the collisions of heavy ions and $e^{+} e^{-}$annihilation can become a laboratory for studying the effects of acceleration, and in the case of annihilation, the effects of acceleration arise in their pure form, unlike ion collisions, where rotation is present at the same time.

One of the most interesting and unusual quantum-field effects of acceleration is the Unruh effect [6], which consists in the appearance of thermal radiation in an accelerated system, with the Unruh temperature $T_{U}=a / 2 \pi$ depending on the acceleration.

Recently it was shown $[7,8]$ that the Unruh effect can be derived by calculation of the quantum corrections from acceleration in the thermodynamic observables. Such corrections in the energymomentum tensor of free massless fermion fields were obtained in [9, 8]. In particular, it was shown that the energy density of a fermion gas moving with acceleration in the laboratory frame is equal to

$$
\rho=\frac{7 \pi^{2} T^{4}}{60}+\frac{T^{2} a^{2}}{24}-\frac{17 a^{4}}{960 \pi^{2}}+\mathscr{O}\left(a^{6}\right)
$$

which is the result of an exact analytical calculation of quantum corrections based on the fundamental equilibrium density operator of Zubarev [10], in which the acceleration effects are described by the term with the boost operator. For instance, the coefficient of the term $A_{2} a^{4}$ of the 4 th order 
in acceleration in (2.1) is obtained by calculating the quantum correlator of the form

$$
A_{2}=\frac{1}{4 !} \int_{0}^{|\beta|} d \tau_{x} d \tau_{y} d \tau_{z} d \tau_{f}\left\langle T_{\tau} \hat{K}_{-i \tau_{x} u}^{3} \hat{K}_{-i \tau_{y} u}^{3} \hat{K}_{-i \tau_{z} u}^{3} \hat{K}_{-i \tau_{f} u}^{3} \hat{T}^{00}(0)\right\rangle_{\beta(x), c}=-\frac{17}{960 \pi^{2}},
$$

where $\hat{K}_{x}$ is a boost operator translated to the vector $x^{\mu}$, and the index $\beta(x), c$ means that averaging is performed by means of the density operator with $a^{\mu}=0$ and only connected correlators are taken.

In [8] it was noted, that (2.1) goes to zero when the proper temperature $T$ is equal to the Unruh temperature

$$
\rho\left(T=\frac{a}{2 \pi}\right)=0 .
$$

Thus, the Minkowski vacuum state corresponds to the proper temperature, measured by the comoving observer, equal to the Unruh temperature, which can be considered as a new derivation of Unruh effect for fermions.

Let's discuss Eq. (2.1) in more detail. Note that (2.1) allows an integral representation in the form of Fermi and Bose integrals

$$
\begin{aligned}
\rho= & \frac{7 \pi^{2} T^{4}}{60}+\frac{T^{2} a^{2}}{24}-\frac{17 a^{4}}{960 \pi^{2}}=2 \int \frac{d^{3} p}{(2 \pi)^{3}}\left(\frac{|\mathbf{p}|+i a}{1+e^{\frac{|\mathbf{p}|}{T}+\frac{i a}{2 T}}}+\frac{|\mathbf{p}|-i a}{1+e^{\frac{|\mathbf{p}|}{T}-\frac{i a}{2 T}}}\right) \\
& +4 \int \frac{d^{3} p}{(2 \pi)^{3}} \frac{|\mathbf{p}|}{e^{\frac{2 \pi \mid \mathbf{p}}{a}}-1} \quad\left(T>T_{U}\right),
\end{aligned}
$$

which coincides exactly with the result of the perturbative calculation if $T>T_{U}$. As shown in [8], the integral formula (2.4) receives additional motivation from the point of view of another approach based on the covariant Wigner function [11].

The plots of the functions (2.4) and (2.1) are shown on the left panel of Fig.1. The difference between the two plots should not be surprising, since it is clear that the perturbative expansion may become non-applicable in the area $a>2 \pi T$.

An interesting consequence of (2.4) is the appearance of acceleration in the form of an imaginary chemical potential, which was also discussed using the example of axial current and Chiral Vortical Effect in [12]. It is interesting to compare this fact with the observation that the angular velocity appears as a real chemical potential [12, 13]. In particular, in [12, 13] (look also [14]) a formula for the axial current of massive fermions was obtained

$$
\begin{aligned}
j_{\mu}^{5}= & \int \frac{d^{3} p}{(2 \pi)^{3}}\left\{n_{F}\left(E_{p}-\mu-\frac{\omega}{2}\right)-n_{F}\left(E_{p}-\mu+\frac{\omega}{2}\right)+\right. \\
& \left.n_{F}\left(E_{p}+\mu-\frac{\omega}{2}\right)-n_{F}\left(E_{p}+\mu+\frac{\omega}{2}\right)\right\} \frac{\omega_{\mu}}{\omega}, \quad \omega=\sqrt{-\omega_{\mu} \omega^{\mu}},
\end{aligned}
$$

reproducing, in particular, the famous Chiral Vortical Effect. In (2.5) $\omega_{\mu}$ is the vorticity and $n_{F}(E)$ is the Fermi distribution.

In [15] the integral (2.4) was transformed to the form of the integral along the contour in the complex plane shown on the right panel of Fig.1. It turns out that the appearance of acceleration as an imaginary chemical potential leads to the rotation of this contour when the acceleration changes and it crosses the Fermi distribution pole $e^{p / T}=-1$ when $T=\frac{a}{2 \pi}$. This cross of the pole leads to 
instability at the Unruh temperature, manifested in the discontinuity of the second order derivative of the energy density

$$
\frac{\partial^{2}}{\partial T^{2}} \rho_{T>T_{U}} \neq \frac{\partial^{2}}{\partial T^{2}} \rho_{T<T_{U}} \quad\left(T \rightarrow T_{U}\right) .
$$

This instability as well as the negativity of energy density below the Unruh temperature initially
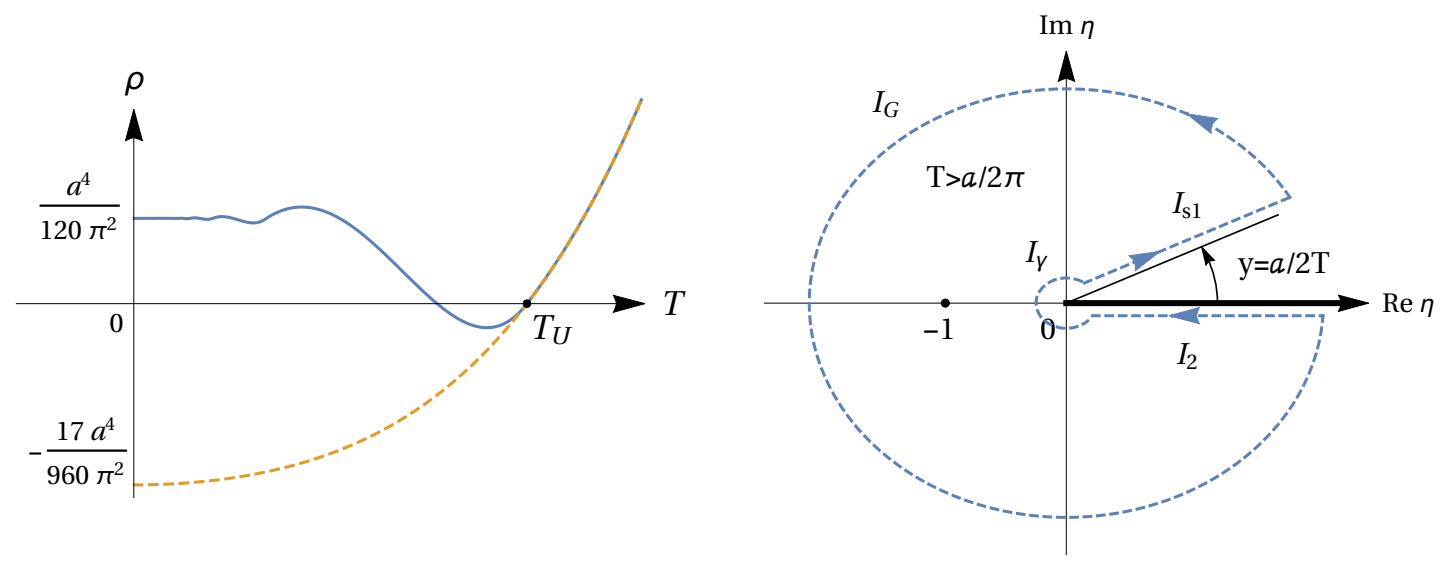

Figure 1: Left: energy density as a function of temperature. The proposed formula (2.4) corresponds to the solid blue line; the perturbative result (2.1), obtained from the density operator, is shown by the dashed orange line. Right: integration contour in integral, to which (2.4) is transformed.

noted in [7], indicate that the Unruh temperature is the lower boundary temperature for the accelerated medium.

Summing up, experimental verification of the expression (2.1) in systems with ultrahigh accelerations could be considered as a way of observing the Unruh effect.

\section{Discussion and Conclusions}

The energy scan at EIC allows one to change the temperature. Combining it with the changing acceleration, by considering various centrality and kinematics of final particles, one can study in detail the Unruh effect.

At the same time, the description of atomic number scan is related to the matching with the QCD description of hadronic Single Spin Asymmetries (SSA) to which polarization belongs.

Their essential feature is the imaginary phase which may be described in QCD due to the interchange of soft quarks [16] and gluons [17] between large and short distances.

The latter case may be related [18] to the time-reversal odd Sivers function [19], whose appearance in QCD is due to the Wilson lines, being another way of describing the soft-hard interactions $[20,21,22]$.

The analog of the imaginary phases generated by these effects was suggested [23] to be the disspation accompanying the transition of angular momentum to spin carried by heavy (baryonic) degrees of freedom in the cores of quantized vortices in the mesonic superfluid. 
Here we would like to point out to the another aspect of this similarity. The memory effect, recently considered [4] for large energy QCD may be naturally attributed just to Sivers function. The gluonic Wilson line, necessarily going to the $t=-\infty$ and back after the transverse kick there may be considered instead as a pair of gluons produced there and coming to the points $x, y$ where they are attached to the quarks entering the respective hadronic correlator. The further study of this description is now under investigation.

To conclude, the energy and atomic number scan at EIC allows one to probe the very finde details of QCD theory and its (sometimes surprising) relations with other branches of physics.

\section{Acknowledgments}

The work was supported in part by RFBR Grants 17-02-01108 and 18-02-01107. O.T. is indebted to Organizers for warm hospitality in Torino. He is also grateful to I. Scimemi and R. Venugopalan for useful discussions.

\section{References}

[1] A. Sorin and O. Teryaev, Phys. Rev. C 95, no. 1, 011902 (2017) doi:10.1103/PhysRevC.95.011902 [arXiv:1606.08398 [nucl-th]].

[2] O. Rogachevsky, A. Sorin and O. Teryaev, Phys. Rev. C 82, 054910 (2010) doi:10.1103/PhysRevC.82.054910 [arXiv:1006.1331 [hep-ph]].

[3] M. Baznat, K. Gudima, A. Sorin and O. Teryaev, Phys. Rev. C 97, no. 4, 041902 (2018) doi:10.1103/PhysRevC.97.041902 [arXiv:1701.00923 [nucl-th]].

[4] A. Ball, M. Pate, A. M. Raclariu, A. Strominger and R. Venugopalan, Annals Phys. 407, 15 (2019) doi:10.1016/j.aop.2019.04.010 [arXiv:1805.12224 [hep-ph]].

[5] P. Castorina, D. Kharzeev and H. Satz, Eur. Phys. J. C 52, 187 (2007) doi:10.1140/epjc/s10052-007-0368-6 [arXiv:0704.1426 [hep-ph]].

[6] W. G. Unruh, Phys. Rev. D 14, 870 (1976). doi:10.1103/PhysRevD.14.870

[7] F. Becattini, Phys. Rev. D 97, no. 8, 085013 (2018) doi:10.1103/PhysRevD.97.085013 [arXiv:1712.08031 [gr-qc]].

[8] G. Y. Prokhorov, O. V. Teryaev and V. I. Zakharov, Phys. Rev. D 99, no. 7, 071901 (2019) doi:10.1103/PhysRevD.99.071901 [arXiv:1903.09697 [hep-th]].

[9] M. Buzzegoli and F. Becattini, JHEP 1812, 002 (2018) doi:10.1007/JHEP12(2018)002 [arXiv:1807.02071 [hep-th]].

[10] D. N. Zubarev, A. V. Prozorkevich, S. A. Smolyanskii, TMF, 40:3 (1979), 394-407; Theoret. and Math. Phys., 40:3 (1979), 821-831.

[11] F. Becattini, V. Chandra, L. Del Zanna and E. Grossi, 'Annals Phys. 338 (2013) 32 doi:10.1016/j.aop.2013.07.004 [arXiv:1303.3431 [nucl-th]].

[12] G. Prokhorov, O. Teryaev and V. Zakharov, Phys. Rev. D 98, no. 7, 071901 (2018) doi:10.1103/PhysRevD.98.071901 [arXiv:1805.12029 [hep-th]].

[13] G. Y. Prokhorov, O. V. Teryaev and V. I. Zakharov, 'JHEP 1902, 146 (2019) doi:10.1007/JHEP02(2019)146 [arXiv:1807.03584 [hep-th]]. 
[14] A. Vilenkin, Phys. Rev. D 21 (1980) 2260. doi:10.1103/PhysRevD.21.2260

[15] G. Y. Prokhorov, O. V. Teryaev and V. I. Zakharov, arXiv:1906.03529 [hep-th].

[16] A. V. Efremov and O. V. Teryaev, Phys. Lett. 150B, 383 (1985). doi:10.1016/0370-2693(85)90999-2

[17] J. w. Qiu and G. F. Sterman, Phys. Rev. Lett. 67, 2264 (1991). doi:10.1103/PhysRevLett.67.2264

[18] X. Ji, J. W. Qiu, W. Vogelsang and F. Yuan, Phys. Rev. Lett. 97, 082002 (2006) doi:10.1103/PhysRevLett.97.082002 [hep-ph/0602239].

[19] D. W. Sivers, Phys. Rev. D 41, 83 (1990). doi:10.1103/PhysRevD.41.83

[20] J. C. Collins, Phys. Lett. B 536, 43 (2002) doi:10.1016/S0370-2693(02)01819-1 [hep-ph/0204004].

[21] A. V. Belitsky, X. Ji and F. Yuan, Nucl. Phys. B 656, 165 (2003) doi:10.1016/S0550-3213(03)00121-4 [hep-ph/0208038].

[22] D. Boer, P. J. Mulders and F. Pijlman, Nucl. Phys. B 667, 201 (2003) doi:10.1016/S0550-3213(03)00527-3 [hep-ph/0303034].

[23] O. V. Teryaev and V. I. Zakharov, Phys. Rev. D 96, no. 9, 096023 (2017). doi:10.1103/PhysRevD.96.096023 